\title{
Article
}

\section{Quantum Switchboard with Coupled-Cavity Array}

\author{
Wai-Keong Mok $^{1}$ D and Leong-Chuan Kwek ${ }^{1,2,3,4,+}$ (D) \\ 1 Centre for Quantum Technologies, National University of Singapore, Singapore 117543, Singapore \\ 2 MajuLab, CNRS-UNS-NUS-NTU International Joint Research Unit, Singapore UMI , Singapore \\ 3 National Institute of Education, Nanyang Technological University, Singapore 637616, Singapore \\ 4 Quantum Science and Engineering Center, Nanyang Technological University, Singapore 637616, Singapore \\ * Correspondence: cqtklc@nus.edu.sg \\ + All authors contributed equally to this work.
}

\begin{abstract}
The ability to control the flow of quantum information deterministically is useful for scaling up quantum computation. In this paper, we demonstrate a controllable quantum switchboard which directs the teleportation protocol to one of two targets, fully dependent on the sender's choice. The quantum switchboard additionally acts as a optimal quantum cloning machine. We also provide a physical implementation of the proposal using a coupled-cavity array. The proposed switchboard can be utilized for the efficient routing of quantum information in a large quantum network.
\end{abstract}

Keywords: quantum entanglement; quantum network; coupled-cavity array

\section{Introduction}

A quantum network contains many quantum nodes for processing and storing quantum states and quantum channels for the distribution of quantum information [1-3]. The ability to distribute arbitrary quantum states is essential for quantum information processing in a quantum network. Efficient navigation in a complex quantum network will eventually need a quantum multiplexer or switchboard to direct the flow of quantum information. A likely scenario is one where we have many quantum devices or computers linked by a switchboard system to other devices and computers for processing, storing or sensing. Many quantum information processes require the explicit preparation of specially entangled quantum states. Two-qubit maximally entangled state often called Bell state, for instance, form an essential quantum resource needed in quantum teleportation [4]. The preparation of three-qubit maximally entangled state (such as GHZ state) could be harnessed for secure secret sharing [5]. In one-way quantum computing, a four-qubit entangled state called cluster state provides an efficient implementation of a universal quantum gate: arbitrary single-qubit unitary operation[6].

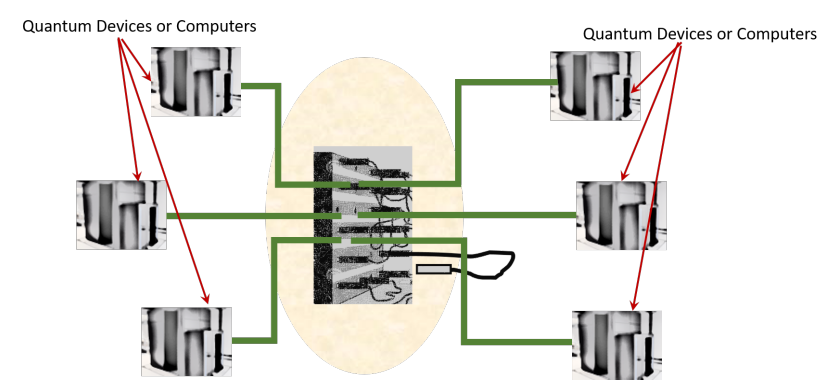

Figure 1. A quantum network consisting of many quantum devices and computers connected through a quantum switchboard. The switchboard can also redirect congested channels to less used channels during peak usage. 
It is interesting to note that entangled states which are used as a common resource in quantum information processes generally need not even be maximally entangled at all. As long as the state is genuinely entangled, quantum computation and communication will generally be better than the classical counterparts. In particular, the non-maximally entangled $\mathrm{W}$ state has been experimentally implemented and proposed for controlled quantum teleportation and secure communication [7].

An essential component of any quantum computation is the ability to spread quantum information over various parts of the quantum computer. The parts then undergo separate evolutions depending on the type of the quantum information processing we wish to implement. Ultimately we need to be capable of navigating the relevant part of the information into a designated output. In a classical computer this flow of information is achieved through a controllable switch. Is it possible to design a quantum analogue for such a device? An added complexity in a quantum switch would be the requirement that the information flows down many possible channels coherently as well as the possibility of channeling it in one selected direction.

Ideally we would like to realize a simple device to achieve this purpose. In addition, these qubits are implemented in practice in a physical system determined by the nature of the qubits and couplings between them. Therefore, in the design of the switch, realistic interaction between the qubits severely limits the type of possible Hamiltonians that can execute such a quantum switch. Here we present a possible implementation of the switch that minimally fulfills these requirements.

In the usual teleportation protocol [8], the sender Alice and receiver Bob begins by sharing a two-qubit maximally entangled Bell state. Alice also possesses an additional ancilla qubit which carries the quantum state to be teleported. Alice then performs a Bell measurement on her share of the entangled state and the ancilla qubit, and the measurement results are then communicated classically to Bob which will perform a corresponding unitary operation on his qubit to recover the desired state, thus completing the teleportation. Note that the choice of receiver is fixed by whoever Alice shares the initial entangled state with, and cannot be changed without discarding the entangled state and setting up a new one with a different receiver Charlene. This poses two limitations to the scheme: In order to teleport quantum states on-demand with multiple receivers, Alice has to share a separate entangled qubit pair with each of the receivers, which must be isolated from one another. Furthermore, Alice is not allowed to choose the receiver after the Bell measurement in this scheme.

In this paper, we show how the above challenges can be overcome by using a specific four-qubit entangled state shared between the sender Alice, the two receivers Bob and Charlene, as well as the accomplice Dick. The role of Dick is to direct the teleported state to the desired receiver as per the sender's choice, which can be done after Alice reveals the Bell measurement results. By controlling the flow of quantum information deterministically, the protocol becomes a quantum switchboard with Dick acting as a 'switchboard operator'. We also provide a physical implementation of the quantum switchboard using a drivencoupled cavity array, which realizes the required multi-partite entangled state.

\section{Controllable quantum switchboard}

The controllable quantum switchboard serves to direct the flow of quantum information in a quantum network [9]. Specifically, it allows Alice to transfer her quantum state perfectly to either Bob or Charlene (free choice) with the help of her accomplice Dick. The basis of the scheme requires all four parties to be initialized in the four-qubit state

$$
|\psi\rangle=\frac{1}{\sqrt{3}}\left(\left|(11)_{A B}\right\rangle\left|(11)_{C D}\right\rangle-\left|(11)_{A D}\right\rangle\left|(11)_{B C}\right\rangle\right)
$$




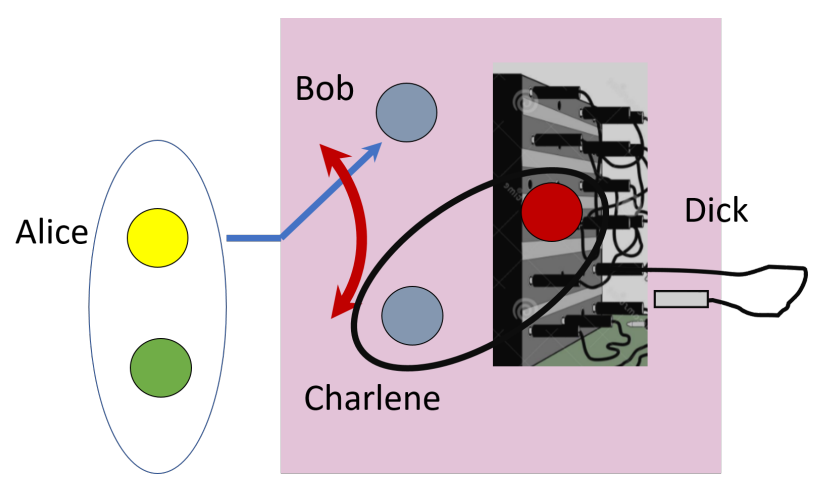

Figure 2. Schematic of the quantum switchboard. Suppose Alice wishes to send her auxiliary qubit to Bob. She can direct Dick to send his qubit to Charlene (Bob). Charlene then performs a Bell measurement on his qubit with Dick's qubit and send the results of his measurement to Bob. Using the information from Charlene, Bob can perfectly recover the state of the Alice's auxiliary qubit.

using the notation for Bell states

$$
|(a b)\rangle=\sum_{k=0}^{1} \frac{(-1)^{k b}}{\sqrt{2}}|k, k \oplus a\rangle
$$

with $\otimes$ denoting modulo-2 addition, or an XOR operation. For example, the state $|(11)\rangle$ represents the singlet state $\frac{1}{\sqrt{2}}(|0\rangle|1\rangle-|1\rangle|0\rangle)$. It turns out that this state is ideally suited for a quantum switchboard, i.e., a circuit that can be used to direct the flow of quantum information in a controllable manner. An interesting property of the presented switchboard is that in the case of failure the information is not entirely lost.

We will now illustrate the quantum switchboard protocol. Suppose Alice wants to teleport her quantum state $|\alpha\rangle_{0}$ encoded in an ancilla qubit (indexed 0) to Bob. The combined state of the ancilla and the initial entangled state can be expressed as

$$
|\alpha\rangle_{0}|\psi\rangle=\frac{1}{4 \sqrt{3}} \sum_{k, l, m, n=0}^{1} \lambda_{k l}\left|(m n)_{0 A}\right\rangle \otimes U_{m n, k l}|\alpha\rangle_{B}\left|(k l)_{C D}\right\rangle
$$

where $\lambda_{11}=3, \lambda_{01}=\lambda_{10}=1$ and $\lambda_{00}=-1 . U_{m n, k l}$ is the unitary transformation on Bob's qubit, determined by the results of both Bell measurements $(m n)$ and $(k l)$. Alice first performs a Bell measurement on the ancilla and her share of $|\psi\rangle$, yielding a measurement result $(\mathrm{mn})$. Immediately after getting one of the four possible outcomes, she broadcasts two (classical) bits of information to Bob and Charlene as it is in the usual teleportation scheme. At this point, it is not necessary for Dick to know these two bits of information.

The Bell measurement collapses the state of the other three parties to be

$$
\left|\chi_{m n}\right\rangle=\sum_{k, l=0}^{1} \frac{\lambda_{k l}}{2 \sqrt{3}} U_{m n, k l}|\alpha\rangle_{B}\left|(k l)_{C D}\right\rangle
$$

If we trace out Charlene and Dick's qubits, then Bob will have the mixed state

$$
\rho_{m n}=\frac{1}{12} \sum_{k, l=0}^{1}\left|\lambda_{k l}\right|^{2} U_{m n, k l}|\alpha\rangle\langle\alpha| U_{m n, k l}^{\dagger}
$$

A similar state is obtained for Charlene if we trace out Bob and Dick's qubits instead. Hence, Bob and Charlene can recover the state of the ancilla qubit with the fidelity $\frac{5}{6}$ by applying appropriate unitary transformation based on the knowledge of the broadcast classical bits. The given state at the beginning does not provide a universal cloning machine for three 
copies of the cloned state [10]. Thus, the qubit belonging to Dick is related to the Alice's ancilla qubit with the "classical" fidelity $\frac{1}{3}$, i.e., the fidelity that can be achieved without prior entanglement. It is interesting to note that Bob and Charlene possess the optimum fidelity achievable under a symmetric cloning machine. Dick's fidelity is allowed since there is no limitation on the production of clones with the fidelity below $\frac{2}{3}$.

In order to obtain perfect fidelity, the accomplice Dick can send his qubit to Charlene to perform Bell measurement, yielding the result $(\mathrm{kl})$. With both measurement results $(m n)$ from Alice and $(k l)$ from Charlene, Bob now has the state

$$
|\phi\rangle_{B}=U_{m n, k l}|\alpha\rangle
$$

Thus, Bob can recover Alice's state $|\alpha\rangle$ (up to a global phase) by simply performing an inverse transform $U_{m n, k l}$ on his qubit. Explicitly, the set of unitaries $U_{m n, k l}$ are the Pauli operators $\{I, X, Y, Z\}$ where $U_{m n, k l}=I$ if $m \oplus k=n \otimes l=0, X$ if $m \oplus k=1$ and $n \oplus l=0$, $Y$ if $m \oplus k=n \otimes l=1$, and lastly $Z$ if $m \oplus k=0$ and $n \oplus l=1$. The presented protocol behaves like an optimal telecloner [11]. However, there is still an unused qubit held by Dick. Depending on Alice's decision regarding to whom she wishes ultimately to send her auxiliary qubit, say Bob (Charlene) for instance, she can direct Dick to send his qubit to Charlene (Bob). as soon as Charlene receives Dick's qubit, he can perform a Bell measurement on his qubit with Dick's qubit and send the results of his measurement to Bob. Using the information from Charlene, Bob can perfectly recover the state of the Alice's auxiliary qubit. Alternatively, Dick can also make the decision on whom he wishes to transmit the unknown qubit held originally by Alice.

The situation is entirely symmetric, i.e., Dick can send his qubit to Bob instead of Charlene with the result that now Charlene can obtain Alice's auxiliary qubit with perfect fidelity. In short, the state acts as a quantum switchboard in which Alice can direct optimal clones to Bob and Charlene or perform perfect quantum teleportation to Bob or Charlene by utilizing Dick's qubit as in a quantum demultiplexer. A schematic diagram of this quantum switchboard protocol is shown in Fig. 2. By directing Dick's qubit to either Bob (or Charlene), Alice can effectively transfer the unknown auxiliary qubit to Charlene (or Bob). Moreover, she can delay the transfer process to a later time as long as she has effective control over Dick's qubit. It is interesting to note that the relative phase between the components of the state $|\psi\rangle$ is crucial for desired functionality. Other phase choices or, for that matter, the complete lack of coherence, will not give us the same quantum switch.

Incidentally, replacing the four-qubit state with a GHZ state, shared among Alice, Bob and Charlene, one could in principle provide perfect quantum teleportation to both Bob and Charlene without the additional benefit of an optimal quantum cloner [12]. In this case, Alice teleclones to both Bob and Charlene with a classical fidelity of $2 / 3$. The eventual quantum teleportation to Bob (or Charlene) is performed with a measurement in the basis $1 / \sqrt{2}(|0\rangle \pm|1\rangle)$. It is also interesting to note that the sheer presence of singlets or dimer-like bonds in the four-qubit state renders it more insensitive to perturbation in the quantum critical regimes. This latter feature is absent in the GHZ state. Let us now prove the above statement. It is convenient to write the state $|\psi\rangle$ in the following way

$$
\begin{aligned}
& |\psi\rangle=\frac{1}{2 \sqrt{3}}\left(3\left|(11)_{12}\right\rangle\left|(11)_{34}\right\rangle+\left|(10)_{12}\right\rangle\left|(10)_{34}\right\rangle+\right. \\
& \left.\left|(01)_{12}\right\rangle\left|(01)_{34}\right\rangle-\left|(00)_{12}\right\rangle\left|(00)_{34}\right\rangle\right) .
\end{aligned}
$$

We can immediately see that the state shared by Alice and Bob is the Werner state with $\frac{1}{3}$ of noise. Taking into account that the fidelity of teleportation for the Werner state with the noise fraction $1-p$ is given by $\frac{p+1}{2}$ [13] we see that the fidelity of Bob's qubit is $\frac{5}{6}$. It can be checked that the state between Alice and Dick is the Werner state that is an equal mixture of the three Bell states $|(10)\rangle,|(01)\rangle,|(00)\rangle$. Thus Dick's clone of Alice's auxiliary qubit has the fidelity $\frac{1}{3}$, which is the fidelity achievable classically. 


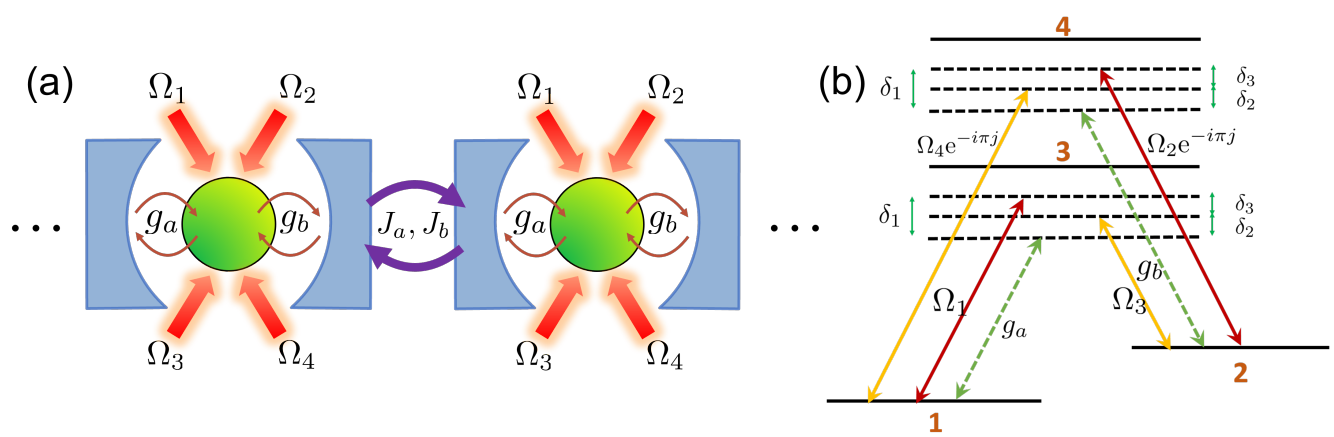

Figure 3. (a) Coupled-cavity array setup. Each unit cell contains a four-level atom coupled to a two-mode cavity field with strengths $g_{a}$ and $g_{b}$. Each atom is driven by four external lasers with Rabi frequencies $\Omega_{1}, \Omega_{2}, \Omega_{3}$ and $\Omega_{4}$. (b) Energy level diagram of the four-level atom. The detunings $\delta_{j}$ are defined in the main text. A double $\Lambda$ system is formed, with $\Lambda_{a}=\{|1\rangle,|2\rangle,|3\rangle\}$ and $\Lambda_{b}=\{|1\rangle,|2\rangle,|4\rangle\}$. The ground states $|1\rangle$ and $|2\rangle$ are used to encode a qubit.

\section{Realization of next nearest-neighbor interactions with coupled-cavity array} [14]

Remarkably, the Majumdar-Ghosh (MG) model given by the spin-chain Hamiltonian

$$
H_{M G}=J \sum_{i=1}^{N}\left(2 \vec{S}_{i} \vec{S}_{i+1}+\alpha \vec{S}_{i} \vec{S}_{i+2}\right)
$$

and periodic boundary conditions is exactly solvable for $\alpha=1$ and even number of spins, with the degenerate ground state manifold spanned by the basis (dimer) states

$$
\begin{aligned}
\left|\psi_{g}\right\rangle & =\left|(11)_{12}\right\rangle\left|(11)_{34}\right\rangle \ldots\left|(11)_{(N-1) N}\right\rangle \\
\left|\phi_{g}\right\rangle & =\left|(11)_{23}\right\rangle\left|(11)_{45}\right\rangle \ldots\left|(11)_{N 1}\right\rangle
\end{aligned}
$$

Thus, the required four-qubit state in Eq. (1) can be prepared from the superposition of the Majumdar-Ghosh Hamiltonian ground states. While the Majumdar-Ghosh model has yet to be realized experimentally, close proximity to the Majumdar-Ghosh point with $\alpha=0.9$ has been experimentally observed in the $\mathrm{Cu}^{2+}$ mineral szenicsite $\mathrm{Cu}_{3}\left(\mathrm{MoO}_{4}\right)(\mathrm{OH})_{4}[15]$, showing the dimerized phase. We also note that both the local and non-local dimers in Eq. ( 9) can be prepared using the dark states of a driven-dissipative spin chain coupled indirectly via mediating photons in a chiral waveguide [16,17]. In this waveguide QED setup, the infinite-range nature of the effective spin-spin interactions give rise to interesting dimer states depending on the detuning pattern of the spins and the chirality of the waveguide. This means that the controllable quantum switchboard can also be potentially realized with a cold-atom chain. Sec. 3 provides a scheme to simulate the physics of the MG model using a driven coupled-cavity array [18].

In this section, we review the proposal introduced in Ref. [18] to realize next nearest neighbor (NNN) spin-chain interactions using a coupled-cavity array, and show how it can be used to implement the quantum switchboard. Consider an array of $N$ cavity QED subsystems. Each subsystem comprises a four-level atom coupled to a bimodal cavity, with 6 optical driving fields. This forms an effective double $\Lambda$ system, with $\Lambda_{a}=\{|1\rangle,|2\rangle,|3\rangle\}$ and $\Lambda_{b}=\{|1\rangle,|2\rangle,|4\rangle\}$ (as shown in Fig. 3). The ground states $|1\rangle$ and $|2\rangle$ will be used as a logical qubit. At the $j^{\text {th }}$ site, the atom is coupled to 2 cavities (modes $a_{j}$ at frequency $\omega_{a}$ and $b_{j}$ at frequency $\omega_{b}$, with coupling strengths $g_{a}$ and $g_{b}$ respectively). The four driving lasers have Rabi frequencies $\Omega_{1}, \Omega_{2} e^{-i \pi j}, \Omega_{3}$ and $\Omega_{4} e^{-i \pi j}$, with laser frequencies $\omega_{1}, \omega_{2}, \omega_{3}$ and $\omega_{4}$ respectively. The transitions are labelled in Fig. 3. We can define the detunings $\delta_{31}=\omega_{31}-\omega_{a}, \delta_{42}=\omega_{42}-\omega_{b}, \Delta_{31}=\omega_{31}-\omega_{1}, \Delta_{42}=\omega_{42}-\omega_{2}, \Delta_{32}=\omega_{32}-\omega_{3}$ and $\Delta_{41}=\omega_{41}-\omega_{4}$. 
Assuming the detunings to be large compared to the Rabi frequencies $\left|g_{a}\right|,\left|g_{b}\right|$, $\Omega_{i}$, we can apply the rotating wave approximation and adiabatically eliminate the excited states $|3\rangle$ and $|4\rangle$. This leads to the Hamiltonian

$$
\begin{aligned}
H & =-\sum_{j}\left[A_{1}\left|1_{j}\right\rangle\left\langle 1_{j}\left|a_{j} e^{i \delta_{1} t}+A_{2}\right| 2_{j}\right\rangle\left\langle 1_{j}\right| a_{j} e^{i \delta_{2} t}+\text { H. c. }\right]-\sum_{j}\left[( - 1 ) ^ { j } B _ { 1 } | 2 _ { j } \rangle \left\langle2_{j}\left|b_{j} e^{i \delta_{1} t}+(-1)^{j} B_{2}\right| 1_{j}\right.\right. \\
& -\sum_{j}\left[A_{3}\left|1_{j}\right\rangle\left\langle 2_{j}\left|e^{i \delta_{3} t}+B_{3}\right| 2_{j}\right\rangle\left\langle 1_{j}\right| e^{i \delta 3 t}+\text { H. c. }\right]-\sum_{j}\left[( \frac { \Omega _ { 1 } ^ { 2 } } { \Delta _ { 3 1 } } + \frac { \Omega _ { 4 } ^ { 2 } } { \Delta _ { 4 1 } } ) | 1 _ { j } \rangle \left\langle1_{j}\left|+\left(\frac{\Omega_{3}^{2}}{\Delta_{32}}+\frac{\Omega_{2}^{2}}{\Delta_{42}}\right)\right| 2_{j}\right.\right. \\
& -\sum_{j}\left[\frac{g_{a}^{2}}{\delta_{31}}\left|1_{j}\right\rangle\left\langle 1_{j}\left|a_{j}^{\dagger} a_{j}+\frac{g_{b}^{2}}{\delta_{42}}\right| 2_{j}\right\rangle\left\langle 2_{j}\right| b_{j}^{\dagger} b_{j}\right]+\sum_{j}\left[J_{a}\left(a_{j}^{\dagger} a_{j+1}+a_{j} a_{j+1}^{\dagger}\right)+J_{b}\left(b_{j}^{\dagger} b_{j+1}+b_{j} b_{j+1}^{\dagger}\right)\right]
\end{aligned}
$$

where the coupling parameters are given by

$$
\begin{array}{ll}
A_{1}=\frac{\Omega_{1} g_{a}}{2}\left(\frac{1}{\Delta_{31}}+\frac{1}{\delta_{31}}\right), & A_{2}=\frac{\Omega_{3} g_{a}}{2}\left(\frac{1}{\Delta_{32}}+\frac{1}{\delta_{32}}\right) \\
A_{3}=\frac{\Omega_{1} \Omega_{3}}{2}\left(\frac{1}{\Delta_{31}}+\frac{1}{\Delta_{32}}\right), & B_{1}=\frac{\Omega_{2} g_{b}}{2}\left(\frac{1}{\Delta_{42}}+\frac{1}{\delta_{42}}\right) \\
B_{2}=\frac{\Omega_{4} g_{b}}{2}\left(\frac{1}{\Delta_{41}}+\frac{1}{\delta_{42}}\right), & B_{3}=\frac{\Omega_{2} \Omega_{4}}{2}\left(\frac{1}{\Delta_{41}}+\frac{1}{\Delta_{42}}\right)
\end{array}
$$

the two-photon detunings are defined as $\delta_{1}=\delta_{31}-\Delta_{31}=\delta_{42}-\Delta_{42}, \delta_{2}=\delta_{31}-\Delta_{32}=$ $\delta_{42}-\Delta_{41}$ and $\delta_{3}=\delta_{1}-\delta_{2}$. $J_{a}$ and $J_{b}$ are the tunneling rates for the NN hopping term between the cavities. The $(-1)^{j}$ factors come from the choice of coupling phases in $\Omega_{2}$ and $\Omega_{4}$. Note that the terms containing $A_{i}$ and $B_{i}$ originate from $\Lambda_{a}$ and $\Lambda_{b}$ respectively (defined in the caption of Fig. 3). Using the ground states $\left|1_{j}\right\rangle$ and $\left|2_{j}\right\rangle$ as qubits, we can define the spin operators $S_{j}^{Z}=\frac{1}{2}\left(\left|2_{j}\right\rangle\left\langle 2_{j}|-| 1_{j}\right\rangle\left\langle 1_{j}\right|\right), S_{j}^{+}=\left|2_{j}\right\rangle\left\langle 1_{j}\left|, S_{j}^{-}=\right| 1_{j}\right\rangle\left\langle 2_{j}\right|$.

In order to simplify the Hamiltonian, we first observe that the third and fourth terms contribute an effective local magnetic field $h_{j} S_{j}^{Z}$ and can be temporarily ignored. The fifth term modifies the two-photon detunings, giving $\delta_{a 1}=\delta_{1}+g_{a}^{2} / \delta_{31}, \delta_{a 2}=\delta_{2}+g_{a}^{2} / \delta_{31}$, $\delta_{b 1}=\delta_{1}+g_{b}^{2} / \delta_{42}$ and $\delta_{b 2}=\delta_{2}+g_{b}^{2} / \delta_{42}$. Assuming periodic boundary conditions, we can take the Fourier transform of $a_{j}$ and $b_{j}$ :

$$
a_{j}\left(b_{j}\right)=\frac{1}{\sqrt{N}} \sum_{k=1}^{N} F_{j k} c_{k}\left(d_{k}\right), \quad F_{j k}=\exp \left(-i \frac{2 \pi}{N} j k\right), \quad \sum_{j=1}^{N} F_{j k}^{*} F_{j l}=N \tilde{\delta}_{k l}
$$

where $\tilde{\delta}_{k l}$ is the Kronecker delta. Going into the rotating frame and assuming large detunings, we can adiabatically eliminate the cavities and obtain (after some algebra)

$$
\begin{aligned}
H & =\sum_{j=1}^{N} 2\left(\frac{J_{a}}{\delta_{a 1}^{2}} A_{1}^{2}-\frac{J_{b}}{\delta_{b 1}^{2}} B_{1}^{2}\right) S_{j}^{Z} S_{j+1}^{Z}+\left(\frac{J_{a}}{\delta_{a 2}^{2}} A_{2}^{2}-\frac{J_{b}}{\delta_{b 2}^{2}} B_{2}^{2}\right)\left(S_{j}^{+} S_{j+1}^{-}+S_{j}^{-} S_{j+1}^{+}\right) \\
& +2\left(\frac{J_{a}^{2}}{\delta_{a 1}^{3}} A_{1}^{2}+\frac{J_{b}^{2}}{\delta_{b 1}^{3}} B_{1}^{2}\right) S_{j}^{Z} S_{j+2}^{Z}+\left(\frac{J_{a}^{2}}{\delta_{a 2}^{3}} A_{2}^{2}-\frac{J_{b}^{2}}{\delta_{b 2}^{3}} B_{2}^{2}\right)\left(S_{j}^{+} S_{j+2}^{-}+S_{j}^{-} S_{j+2}^{+}\right)+\mathcal{O}\left(\frac{J_{\mu}^{3}}{\delta_{\mu i}^{3}}\right)
\end{aligned}
$$

for $\mu=a, b$ and $i=1,2$. The first two terms correspond to the NN interactions and the last two terms correspond to the NNN interactions. We can rewrite the above spin-chain Hamiltonian (with local magnetic fields) in a more compact form

$$
H=\sum_{j=1}^{N} \sum_{i=1}^{2} J_{i}\left(S_{j}^{X} S_{j+i}^{X}+S_{j}^{Y} S_{j+i}^{Y}\right)+\lambda_{i} S_{j}^{Z} S_{j+i}^{Z}+h_{j} S_{j}^{Z}
$$


where

$$
\begin{aligned}
& J_{1}=2\left(\frac{J_{a}}{\delta_{a 2}^{2}} A_{2}^{2}-\frac{J_{b}}{\delta_{b 2}^{2}} B_{2}^{2}\right) \\
& \lambda_{1}=2\left(\frac{J_{a}}{\delta_{a 1}^{2}} A_{1}^{2}-\frac{J_{b}}{\delta_{b 1}^{2}} B_{1}^{2}\right)
\end{aligned}
$$

are the NN interaction strengths, and

$$
\begin{aligned}
& J_{2}=2\left(\frac{J_{a}^{2}}{\delta_{a 2}^{3}} A_{2}^{2}+\frac{J_{b}^{2}}{\delta_{b 2}^{3}} B_{2}^{2}\right) \\
& \lambda_{2}=2\left(\frac{J_{a}^{2}}{\delta_{a 1}^{3}} A_{1}^{2}+\frac{J_{b}^{2}}{\delta_{b 1}^{2}} B_{1}^{2}\right)
\end{aligned}
$$

are the NNN interaction strengths. Controlling the parameters appropriately, the MG model in Eq. (8) can be realized.

Note that the scheme cannot work without both $\Lambda_{a}$ and $\Lambda_{b}$. If we only consider the $\Lambda_{a}$ system, the NNN interaction strengths will be weaker than the NN interaction strengths by an order of $J / \delta$. Since we require $\delta$ to be large, the NNN strengths will thus be very weak and cannot realize the MG Hamiltonian. With the two $\Lambda$ systems, the driving phase can then produce the $(-1)^{j}$ factors in Eq. (10), which can be utilized to suppress NN interactions and boost NNN interactions (notice the sign differences between Eq. (15) and Eq. (16) ). As such, the resultant NN and NNN interaction strengths can be made comparable by controlling the free laser and cavity parameters.

In order to obtain the MG Hamiltonian, we require $J_{1}=\lambda_{1}, J_{2}=\lambda_{2}$ and $J_{1}=2 J_{2}$. This can be realized (for example) by setting $\delta_{1}=\delta_{2}, \Omega_{1}=\Omega_{3}, \Omega_{2}=\Omega_{4}, \Delta_{31}=\Delta_{32}, \Delta_{41}=\Delta_{42}$, $\delta_{31}=\delta_{32}, J_{a}=J_{b}, \delta_{a 2}=\delta_{b 2}, B_{2}=A_{2} / 2, J_{a}=0.3 \delta_{a 2}$. As demonstrated in [18], the $J_{1} / J_{2}$ ratio can be adjust arbitrarily and can even be greater than 1 , even though each term in $J_{1}$ is significantly larger than $J_{2}$. Moreover, the qubit is encoded in the ground states of the four-level atom, it is highly robust against decoherence due to dissipation. There is also negligible transitions to the excited states during the process due to large detunings, which justifies the adiabatic elimination of excited states.

\section{Conclusion}

In conclusion, we demonstrate the controllable quantum switchboard which directs the flow of quantum information in a quantum network, by allowing the sender to freely choose one of the two targets to receive the quantum state via teleportation. The key difference with other controlled teleportation protocols is that in the event of failure, the quantum information is not entirely lost and the switchboard behaves as an optimal telecloner where the receivers can recover the quantum state with a fidelity of $\frac{5}{6}$. We also show the physical implementation of the initial four-qubit entangled state (as the ground state of the Majumdar-Ghosh model) using a coupled-cavity array. The quantum switchboard can potentially be a useful component of a large quantum network, where the information pathway can be redirected efficiently to reduce network congestion.

\section{References}

1. Kimble, H.J. The quantum internet. Nature 2008, 453, 1023-1030.

2. Wehner, S.; Elkouss, D.; Hanson, R. Quantum internet: A vision for the road ahead. Science 2018, 362.

3. Lloyd, S.; Shapiro, J.H.; Wong, F.N.; Kumar, P.; Shahriar, S.M.; Yuen, H.P. Infrastructure for the quantum Internet. ACM SIGCOMM Computer Communication Review 2004, 34, 9-20.

4. Bennett, C.H.; Brassard, G.; Crépeau, C.; Jozsa, R.; Peres, A.; Wootters, W.K. Teleporting an unknown quantum state via dual classical and Einstein-Podolsky-Rosen channels. Physical review letters 1993, 70, 1895.

5. Gottesman, D. Theory of quantum secret sharing. Physical Review A 2000, 61, 042311.

6. Raussendorf, R.; Briegel, H.J. A one-way quantum computer. Physical Review Letters 2001, 86, 5188. 
7. Eibl, M.; Kiesel, N.; Bourennane, M.; Kurtsiefer, C.; Weinfurter, H. Experimental realization of a three-qubit entangled W state. Physical review letters 2004, 92, 077901.

8. Bennett, C.H.; Brassard, G.; Crépeau, C.; Jozsa, R.; Peres, A.; Wootters, W.K. Teleporting an unknown quantum state via dual classical and Einstein-Podolsky-Rosen channels. Phys. Rev. Lett. 1993, 70, 1895-1899. doi:10.1103/PhysRevLett.70.1895.

9. Kaszlikowski, D.; Kwek, L.C.; Lai, C.H.; Vedral, V. Controllable Quantum Switchboard, 2007, [arXiv:0704.1280].

10. Scarani, V.; Iblisdir, S.; Gisin, N.; Acin, A. Quantum cloning. Reviews of Modern Physics 2005, 77, 1225.

11. Murao, M.; Jonathan, D.; Plenio, M.; Vedral, V. Quantum telecloning and multiparticle entanglement. Physical Review A 1999, $59,156$.

12. Karlsson, A.; Bourennane, M. Quantum teleportation using three-particle entanglement. Phys. Rev. A 1998, 58, 4394-4400. doi:10.1103/PhysRevA.58.4394.

13. Horodecki, M.; Horodecki, P.; Horodecki, R. General teleportation channel, singlet fraction, and quasidistillation. Phys. Rev. A 1999, 60, 1888-1898. doi:10.1103/PhysRevA.60.1888.

14. Kumar, B. Quantum spin models with exact dimer ground states. Phys. Rev. B 2002, 66, 024406. doi:10.1103/PhysRevB.66.024406.

15. Lebernegg, S.; Janson, O.; Rousochatzakis, I.; Nishimoto, S.; Rosner, H.; Tsirlin, A.A. Frustrated spin chain physics near the Majumdar-Ghosh point in szenicsite $\mathrm{Cu}_{3}\left(\mathrm{MoO}_{4}\right)(\mathrm{OH})_{4}$. Phys. Rev. B 2017, 95, 035145. doi:10.1103/PhysRevB.95.035145.

16. Ramos, T.; Pichler, H.; Daley, A.J.; Zoller, P. Quantum Spin Dimers from Chiral Dissipation in Cold-Atom Chains. Phys. Rev. Lett. 2014, 113, 237203. doi:10.1103/PhysRevLett.113.237203.

17. Pichler, H.; Ramos, T.; Daley, A.J.; Zoller, P. Quantum optics of chiral spin networks. Phys. Rev. A 2015, 91, 042116. doi:10.1103/PhysRevA.91.042116.

18. Chen, Z.X.; Zhou, Z.W.; Zhou, X.; Zhou, X.F.; Guo, G.C. Quantum simulation of Heisenberg spin chains with next-nearest-neighbor interactions in coupled cavities. Phys. Rev. A 2010, 81, 022303. doi:10.1103/PhysRevA.81.022303. 